\title{
Effect of randomness on ferroelastic transitions: Disorder-induced hysteresis loop rounding in $\mathrm{Ti}-\mathrm{Nb}-\mathrm{O}$ martensitic alloy
}

\author{
Yoichi Nii, ${ }^{1,2}$ Taka-hisa Arima, ${ }^{1}$ Hee Young Kim, ${ }^{2}$ and Shuichi Miyazaki ${ }^{2}$ \\ ${ }^{1}$ Institute of Multidisciplinary Research for Advanced Materials, Tohoku University, Sendai 980-8577, Japan \\ ${ }^{2}$ Institute of Materials Science, University of Tsukuba, Tsukuba 305-8573, Japan \\ (Received 27 August 2010; published 8 December 2010; publisher error corrected 17 December 2010)
}

\begin{abstract}
We have studied the effect of disorder on the martensitic transformation (MT), especially on the hysteretic behavior of Ti-Nb-O alloy. This system allows arbitrary tuning of the amount of disorder by the introduction of interstitial oxygen atoms into elastically soft host lattice [Ti-26Nb (at. \%) alloy], which results in the formation of randomly oriented nanometer-sized martensite as confirmed by dark-field transmission electron microscopy images. In the clean system without interstitial oxygen doping, we observe a sharp hysteresis loop with plateau region in both a stress-strain curve and a strain-temperature curve, whereas a slim hysteresis loop without plateau appears by introducing disorder. The rounding of hysteretic behavior can be understood in random-field Ising model regime, providing the possibility of a new physical picture for MT in a dirty system, where the small size of martensite homogeneously nucleates and percolates the whole system.
\end{abstract}

DOI: 10.1103/PhysRevB.82.214104

PACS number(s): 62.20.fg, 61.05.J-, 61.43.-j

\section{INTRODUCTION}

Martensitic transformation (MT) is a first-order diffusionless structural phase transition. ${ }^{1}$ It shows up as an abrupt jump of the order parameter (strain) at a transition point with a temperature change or the application of stress. MT has been extensively studied for many years because of its importance in the technological applications as shape-memory or superelastic materials. Ordinary MT process can be characterized by the nucleation and growth of low-symmetry martensite phase in high-symmetry parent phase as a collective motion of local events called domino reaction or avalanche $^{2-6}$ The martensite, a kind of ferroelastic, has a lot of physical analogies to other ferroic systems such as ferromagnetic and ferroelectric with magnetic and electric polarizations being the order parameters, respectively. For these ferroic systems, disorder or randomness provides rich physics. Glassiness is a typical example, studied intensively in the past decades. Spin glass in ferromagnetics, ${ }^{7}$ relaxor in ferroelectrics, ${ }^{8}$ and recently discovered strain glass (SG) in ferroelastic martensites ${ }^{9-14}$ share common signature of glass. For example, they exhibit a frequency-dependent anomaly in the response function (magnetic susceptibility, dielectric constant, and strain susceptibility, respectively) and history dependence of the order parameter on whether field-cooling or zero-field-cooling process. These glassy characteristics should correspond to the kinetic (or dynamic) aspect of these disordered ferroic systems. In the static aspect, on the other hand, what influence does disorder exert on ferroic systems? Here the word static means athermal in which thermal fluctuations play an irrelevant role: as the system often lies in some metastable state separated by large energy barriers in the free-energy landscape, relaxation toward the equilibrium state becomes inaccessible on experimental time scale. In this case, thermal activation is negligible (viz., athermal) and consequently, a nonequilibrium hysteretic behavior emerges in the response to an external field. In addition, as the presence of disorder leads to a formation of an exponential number of metastable states in the free-energy landscape, the resultant hysteretic behavior can be modified, ${ }^{2,15-20}$ where the hysteresis serves as a foot print of the complexity of its free-energy landscape. Therefore, the hysteretic behavior in disordered ferroic system is an intriguing subject to study.

A number of spin-based models have been investigated for a first-order phase transition in the presence of random fields (RFs). ${ }^{2,16-24}$ A main difference among them is the number of the degrees of freedom; each spin variable $s_{i}$ has two states $\left(s_{i}=+1,-1\right)$ in Ising-type model, ${ }^{2,16-22}$ three states $\left(s_{i}=+1,0,-1\right)$ in Blume-Emery-Griffith-type model, ${ }^{23}$ and an arbitrary number of states in Potts-type model, ${ }^{24}$ respectively. Thus, when the system transforms into energetically equivalent states, like a martensitic system which has several degenerate domains (or variants), RF Potts model (RFPM) (Ref. 24) is considered to be most suitable. However, considering a competition between only two states (the parent phase and the most relevant domain in martensite phase), we can employ an Ising-type Hamiltonian as a minimal model for MT, where $\left\langle s_{i}\right\rangle=-1,+1$ correspond to a parent and martensite single domain state, respectively. Here we use a slightly modified model of zero-temperature RF Ising model (RFIM); a soft-spin version of zero-temperature RFIM. ${ }^{17,25}$ This model is simple but can extract the essence of the effect of randomness on MT. In particular, the spin $s_{i}$ can change continuously in a double-well potential, taking the classical nature of the lattice strain in consideration. The potential expressed as $V\left(s_{i}\right)=k\left(\left|s_{i}\right|-1\right)^{2}$ has minima at $s_{i}=-1$ (the parent phase) and $s_{i}=+1$ (martensite). In this model, a random field $h_{i}$ with a Gaussian distribution $\rho(h)=\exp ($ $\left.-h^{2} / 2 R^{2}\right) /(\sqrt{2 \pi} R)$ is added at each site, interacting with each Ising spin. The standard deviation $R$ characterizes the disorder strength. Then the model Hamiltonian is described as

$$
\mathcal{H}=-\sum_{\langle i, j\rangle} J_{i j} s_{i} s_{j}-\sum_{i} h_{i} s_{i}-H \sum_{i} s_{i}+\sum_{i} V\left(s_{i}\right) .
$$

Here $H$ is the uniform magnetic field. Each spin ferromagnetically interacts to all the other spins with the coupling constants $J_{i j}(>0)$. Figure 1 shows a mean-field calculation of 


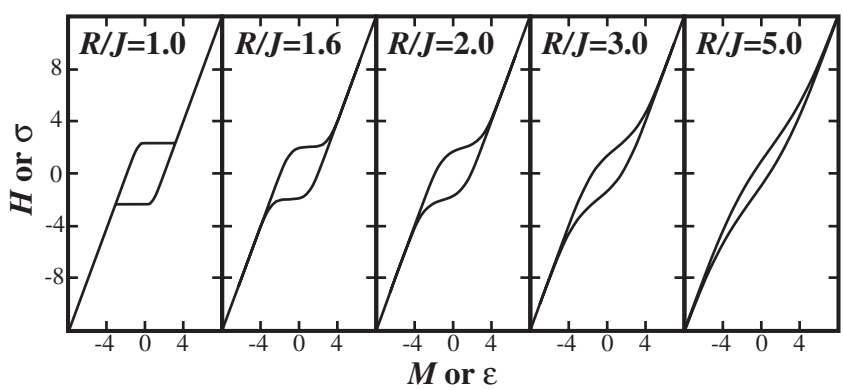

FIG. 1. Mean-field calculation of hysteresis loops with various disorder $(R)$ in soft-spin RFIM (Refs. 17 and 25). Here, we set $J$ $=1$ and $k=2$. $H$ (magnetic field) and $M$ (magnetization) are replaced by $\sigma$ (stress) and $\varepsilon$ (strain), respectively, for a martensitic system.

hysteresis loops in a soft-spin version of RFIM with various disorder $R$ up to considerably strong disorder regime $R / J$ $\gg R_{\mathrm{c}} / J \sim 1.6\left(R_{\mathrm{c}}\right.$ indicates the critical disorder at which critical phenomena arise $\mathrm{e}^{2,17}$ ). With increasing disorder, a variation in hysteresis loop from a sharp and wide to a round and slim one can be seen. In a ferromagnetic system, the change can be understood by the following scenario. ${ }^{2,16,20}$ In a fairly clean system as in the case of $R / J=1.0$, if a domain nucleates by applying an external magnetic field, it grows until the domain wall reaches the edge of the sample as an infinite avalanche. As a result, the magnetization curve has discontinuous jumps at the coercive magnetic fields. In a dirty system as in the case of $R / J=3.0$, in contrast, the presence of strong 'RFs' causes two characteristic changes from the clean case. First, when sweeping the magnetic field from a large negative value to a positive value, even at a negative magnetic field, an up-spin domain is nucleated in some region in a strong positive random field $+h_{i}$, since the effective field becomes positive. Thus, $M-H$ curves of a dirty system have some large gradient even below the apparent coercive magnetic field. Second, even if the magnetization of a domain flips up by applying an external magnetic field, the domain does not grow as an infinite avalanche. The flipped domain will soon reach a place with negative $\mathrm{RFs}-h_{i}$, which pins the domain wall not to move. By further increasing the magnetic field, another domain can flip where the total effective field changes its sign, although the domain growth should also be bound immediately by the other disorder. In this way, the magnetization reversal process has no macroscopic discontinuity corresponding to an infinite avalanche. Instead, it should be composed of a multitude of microscopic discontinuities corresponding to a great number of small avalanches, leading to a slim hysteresis loop. If the RFs are so strong that all avalanches are bound, the phase transition will become macroscopically homogeneous where randomly generated single domain flips percolate the whole system.

A similar situation may occur in a physically parallel system, ferroelastic martensite, but with the possibility of a new physical picture for MT: ordinary MT picture, or nucleation and growth, corresponds to the physical picture of weak disorder regime in RFIM. If the strong disorder suppresses the growth of martensite, in contrast, only the homogeneous nucleation of small-sized martensite can be the main mechanism, and these quite small domains (for example, nanometer-sized martensite) homogeneously percolate the whole system via a multitude of small avalanches.

In this paper, we explain why Ti-Nb-O alloy can be a representative system for the application of RFIM, where interstitial oxygen atoms would act as effective RFs for martensitic system as observed by dark-field transmission electron microscopy (TEM) images, and that the observed disorder-induced hysteresis loop variation in this system is in qualitative agreement with that predicted in RFIM. These correspondences indicate the new MT picture in strongly disordered martensitic system.

\section{EXPERIMENTAL}

Oxygen-doped Ti-Nb-O shape memory alloy ${ }^{26,27}$ was selected as a disordered martensitic system, whereas well studied disordered system is a nonstoichiometric $\mathrm{Ti}_{48.5}-\mathrm{Ni}_{51.5}$ strain glass ${ }^{9-14}$ because of the following reasons. The main difference between $\mathrm{Ti}-\mathrm{Nb}-\mathrm{O}$ and Ti-Ni systems is the type of disorder; interstitial atoms in $\mathrm{Ti}-\mathrm{Nb}-\mathrm{O}$ while substitutional atoms in Ti-Ni. Because the interstitial atom more significantly distorts the surrounding local structure than the substitutional atom does, the introduction of oxygen atoms in the Ti-Nb system allows us to perform experiment up to comparatively strong disorder regime. In addition, the strain field induced by interstitial atoms is simpler to understand than that by substitutional atoms. Each interstitial atom is known to induce local strain ellipsoid. ${ }^{28}$

The Ti-26Nb- $x \mathrm{O}$ (at. \%) $(x=0,0.3,0.5,1.0)$ alloys were fabricated by the Ar-arc melting method from the constituents $\mathrm{Ti}, \mathrm{Nb}$, and $\mathrm{TiO}_{2}$. The Ti-26Nb (at. \%) host alloy has $T_{\mathrm{c}}^{*}$ (martensitic transformation start temperature) around $280 \mathrm{~K}$, leading to elastically soft host lattice and the oxygen concentration was controlled by adding $\mathrm{TiO}_{2}$. Hereafter, each alloy is called by its oxygen concentration like $0 \mathrm{O}, 0.3 \mathrm{O}$, and so on. The ingots were cold rolled to plates of $0.125 \mathrm{~mm}$ with a reduction ratio up to $98.5 \%$. The cold-rolled plates were cut using an electrodischarge machine. The specimens were heat treated at $1173 \mathrm{~K}$ for $1.8 \mathrm{ks}$ in quartz tubes filled with Ar gas, and subsequently quenched into water by breaking the quartz tubes. In order to confirm that the interstitial oxygen atoms act as effective random fields for martensitic system, a TEM observation was carried out at room temperature using JEOL $2010 \mathrm{~F}$ at $200 \mathrm{kV}$. The specimens for TEM observation were punched out from heat-treated specimen after reducing the thickness to below $80 \mu \mathrm{m}$ by chemical etching with a solution containing $\mathrm{HF}, \mathrm{HNO}_{3}$, and $\mathrm{H}_{2} \mathrm{O}$ (1:4:5). Then, the final thinning of observation area was carried out using a conventional twin-jet polishing technique with a solution of $\mathrm{HF}$, $\mathrm{H}_{2} \mathrm{SO}_{4}$, and $\mathrm{CH}_{3} \mathrm{OH}(2: 5: 93)$ at about $233 \mathrm{~K}$. In the case of $\mathrm{Ti}-\mathrm{Nb}$ system, the orthorhombic martensite structure has a twofold modulation along the $\langle 110\rangle$ direction [see Figs. $3(\mathrm{~b})-3(\mathrm{~g})]$, which results in the formation of $\left\{h+\frac{1}{2} k+\frac{1}{2} 0\right\}$ reciprocal lattice points and contributes to superlattice spots. Thus, dark-field images were obtained from the $\left(\frac{3}{2} \frac{1}{2} 0\right)$ superlattice spot for various amounts of oxygen. The stress-strain and strain-temperature hysteresis loops were measured by tensile testing at several temperatures and thermal cycling under several stresses, respectively. 


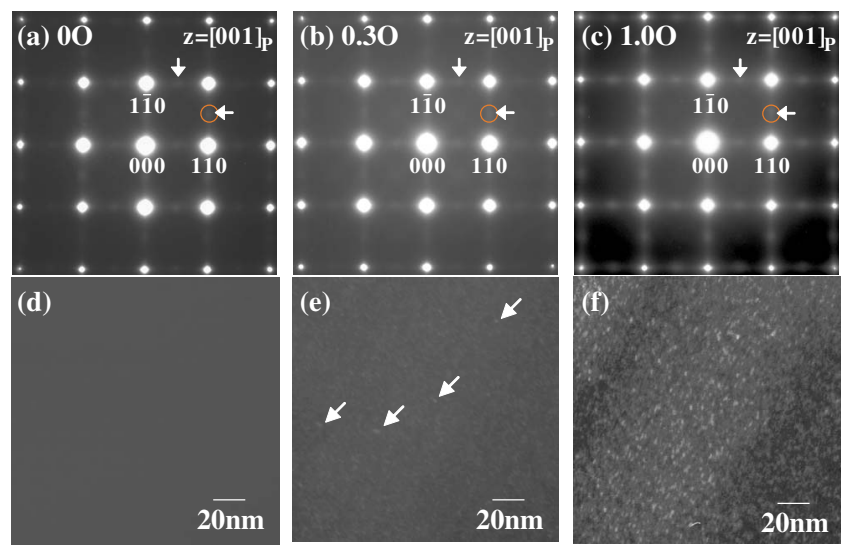

FIG. 2. (Color online) (Top) Electron-diffraction patterns at $[001]_{\mathrm{P}}$ zone axis of Ti-26Nb- $x \mathrm{O}$ (at. \%) alloys with the several amounts of disorder (a) $x=0$, (b) $x=0.3$, and (c) $x=1.0$. (Bottom) Corresponding dark-field images (d)-(f) with a superlattice spot $\left(\frac{3}{2} \frac{1}{2} 0\right)$ surrounded by a circle in the diffraction pattern. The amount of nanometer-sized domains increases with increasing oxygen content, indicating the coupling between disorder (oxygen) and order parameter (strain). This suggests that oxygen atoms act as effective random stress fields (see text).

\section{RESULTS AND DISCUSSIONS}

\section{A. Interstitial oxygen atoms as a source of effective random stress fields for martensite}

Figures 2(a)-2(c) show electron-diffraction patterns of the parent phase of $\mathrm{Ti}-26 \mathrm{Nb}-x \mathrm{O}$ (at. \%) alloys with the amounts of disorder $x=0, x=0.3$, and $x=1.0$ at room temperature. From an X-ray diffraction (XRD) measurement, all the samples are confirmed to be of the parent bcc phase at room temperature (not shown). Nevertheless, one can see superlattice spots at $\left\{h+\frac{1}{2} k+\frac{1}{2} 0\right\}$ and diffuse scattering along $\langle 110\rangle$ directions in the electron-diffraction patterns. The bottom figures represent the dark-field images from the $\left(\frac{3}{2} \frac{1}{2} 0\right)$ superlattice spot surrounded by circles in the diffraction patterns. In the diffraction pattern for the $0 \mathrm{O}$ alloy shown in Fig. 2(a), both the superlattice reflections at $1 / 2\langle 110\rangle$ indicated by arrows and the diffuse scattering in $\langle\xi \xi 0\rangle$ directions around Bragg spots are very weak. With increasing the amount of disorder, however, the intensities of both the superlattice reflections and the diffuse scattering become stronger. In the corresponding dark-field image of $0 \mathrm{O}$ alloy [Fig. 2(d)], there is almost no contrast, indicating the uniform cubic state. By doping 0.3 at. \% oxygen, there appears nanometer-sized regions (nanodomains) indicated by arrows in Fig. 2(e). By further increasing the amount of disorder up to 1.0 at. $\%$ [Fig. 2(f)], the nanodomains grow in size and number, and spread throughout the specimen. In many other martensitic systems, similar microstructures appear as a nanodomain ${ }^{9,29}$ like observed here or a mottled tweed structure ${ }^{30}$ (nanoscale texture of the lattice strain) observed in Ni-Al (Ref. 31) and Fe-Pd (Ref. 32) shape memory alloys. [Which of nanoscale structures appears strongly depends on the elastic anisotropy factor $\left.A=c_{44} /\left(c_{11}-c_{12}\right) .{ }^{33}\right]$ These are known as precursor phenomena prior to MT, where the nanoscale structure is considered to be a local martensitelike region with breaking

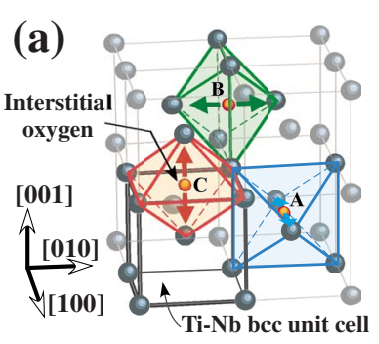

(b)

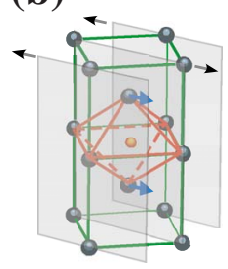

(d)

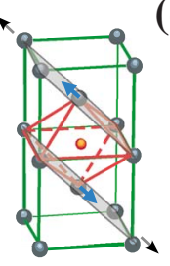

(e)

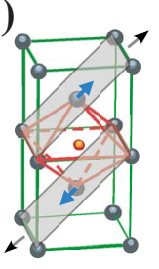

(f)

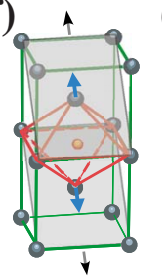

(c)

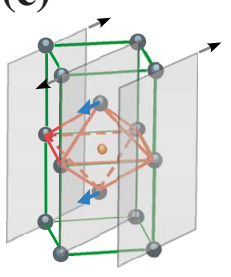

(g)

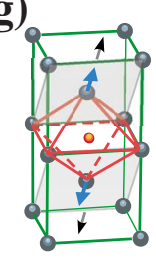

FIG. 3. (Color online) (a) Schematic illustration of three types of octahedral interstitial sites A, B, and C in a bcc lattice. Interstitial atoms at octahedral sites $\mathrm{A}, \mathrm{B}$, and $\mathrm{C}$ are defects with tetragonal symmetry, locally producing ellipsoidal elastic distortion (elastic dipole) along the tetragonal axis [100], [010], and [001], respectively. Random distribution of interstitial oxygen atoms at the three types of octahedral sites A, B, and C would results in the formation of random 'stress' fields in bcc host lattice. [(b)-(g)] Six types of shuffling modes which corresponds to the six orthorhombic martensite domains. When an oxygen atom occupies the interstitial site $\mathrm{C}$, the distance between the nearest-neighbor atoms from the oxygen is relatively elongated along the tetragonal axis [001] by the shuffling modes (d)-(g), whereas the shuffling modes (b) and (c) keep the distance constant. This indicates that the shuffling mode $(\mathrm{d})-(\mathrm{g})$ would be preferential when an oxygen atom occupies the $\mathrm{C}$ site, suggesting the coupling of the interstitial atoms and the martensitic distortion, or the shuffling.

higher symmetry of the parent phase. In a similar manner, the faint diffuse scattering and superlattice reflections of $0 \mathrm{O}$ alloy at room temperature [Fig. 2(a)] may be assigned to the precursor phenomena prior to MT because the observation temperature is close to $T_{\mathrm{c}}^{*}$ for $0 \mathrm{O}$ alloy $(\sim 280 \mathrm{~K})$. However, the observed increase in the amount of nanodomains by oxygen doping cannot be ascribed to the thermal fluctuation because disorder reduces the $T_{\mathrm{c}}^{*}$ value. This decrease in $T_{\mathrm{c}}^{*}$ should make precursor phenomena less efficient in oxygendoped $0.3 \mathrm{O}$ and $1.0 \mathrm{O}$ alloys than pure alloy. Thus, it is strongly suggested that the confirmed local structures should be oxygen-induced nanodomains.

In the bcc lattice, interstitial impurity atoms can occupy the tetrahedral or octahedral sites. For example, carbon in bcc Fe occupies the octahedral sites, whereas the tetrahedral site is assumed to be a transition state between two minima of octahedral sites. ${ }^{34}$ A calculation using the embedded-cell model found that oxygen in Ti-Nb alloy also prefers to occupy the octahedral sites. ${ }^{35}$ This model successfully reproduces the experimentally observed internal friction (Snoek peak). Therefore, we assume that the interstitial oxygen atoms in $\mathrm{Ti}-26 \mathrm{Nb}$ (at. \%) bcc alloy only occupy the octahedral sites. Figure 3(a) shows three types of octahedral sites which interstitial oxygen atoms can occupy in the Ti-Nb bcc lattice. Each octahedral site is surrounded by six atoms, two 
of which are in the shorter $\langle 001\rangle$ direction and four in the longer $\langle 110\rangle$ directions, being $a / 2$ and $a / \sqrt{2}$ from the center of octahedron, respectively. Therefore, the octahedral interstitial atom is a defect with tetragonal local symmetry. Each defect produces local strain ellipsoid, 'elastic dipole' which is characterized by second-rank strain tensor $\lambda$ having only diagonal components with principal values $\lambda_{1}=\lambda_{2}$ and $\lambda_{3}$ along three principal axes. ${ }^{28}$ Oxygen atom at $\mathrm{C}$ site in Fig. 3(a), for example, yields elongation strain along the tetragonal axis [001] as depicted by arrows. For the A and B sites, the tetragonal axis is parallel to [100] and [010], respectively. As a result, there are three types of elastic dipoles corresponding to the interstitial oxygen at $\mathrm{A}, \mathrm{B}$, and $\mathrm{C}$ sites. If the system is in thermal equilibrium and without external stress, three types of elastic dipoles, resulting from the random distribution of interstitial atoms at octahedral sites, should be the same in quantity and distribute spatially at random. Thus in the case of Ti-Nb-O alloy, it is conceivable that randomly oriented elastic dipoles are randomly embedded in the bcc host lattice, which results in the formation of random stress fields in the system.

Here we discuss the effect of the elastic dipoles on MT in detail. In a Ti-Nb alloy, MT is accompanied by staggered shear of $\{1 \overline{1} 0\}$ basal plane along $\langle 110\rangle$ direction, which is called shuffling. There are six shuffling modes corresponding to six kinds of orthorhombic martensite domains. Figures $3(\mathrm{~b})-3(\mathrm{~g})$ show the six shuffling modes with an oxygen atom located at an octahedral $\mathrm{C}$ site. Arrows indicate the shear direction by shuffling. With the shuffling mode in Fig. 3(d), for example, the distance between the nearest-neighbor atoms from oxygen is relatively elongated along the tetragonal axis [001]. This indicates that MT can release the elastic energy produced by the insertion of oxygen atoms. The elongation strain along the tetragonal axis of the octahedron is estimated to be about $+10 \%$ from the atomic displacement by shuffling of Ti-20Nb (at. \%) alloy. ${ }^{36}$ Note that this elongation strain is considerably larger than the Bain strain (lattice distortion along the principle axes), which is at most $4 \%$ in the case of Ti-Nb shape memory alloy. ${ }^{26}$ The shuffling can hence play a central role in relaxing the elastic strain energy. So, if the system is near $T_{\mathrm{c}}^{*}$ and has an elastically 'soft' host lattice against shear along $\langle 110\rangle$ on $\{1 \overline{1} 0\}$, the insertion of elastic dipoles will result in the spontaneous formation of local martensitelike domains. In addition, the positions which oxygen atoms occupy have a profound influence on which shuffling modes are preferentially induced. The six shuffling modes can be divided into two groups for the oxygen atom at $\mathrm{C}$ site, where the tetragonal axis of elastic dipole is in-basal plane [Figs. 3(b) and 3(c)] or out-of-basal plane [Figs. 3(d) $-3(\mathrm{~g})]$, respectively. The shuffling mode with the tetragonal axis in-basal plane has no effect on the distance between the nearest-neighbor atoms from oxygen. In other words, these two shuffling modes are inefficient in releasing its elastic energy. On the other hand, the shuffling modes with the tetragonal axis out-of-basal plane cause relative elongation of the octahedron along the tetragonal axis. Thus these four shuffling modes are preferred when the oxygen atom is at $\mathrm{C}$ site. In the same way, if the tetragonal axis is along [100] (A site) or [010] (B site), the shuffling modes of

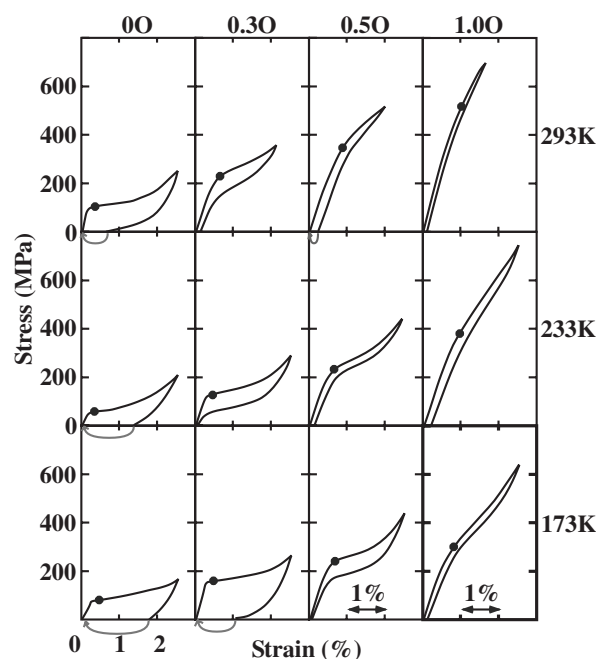

FIG. 4. Stress-strain curves of Ti-26Nb- $x \mathrm{O}$ (at. \%) alloys $(x$ $=0,0.3,0.51 .0)$ at several temperatures. The hysteresis loop varies from a sharp and wide loop to a round and slim one with increasing disorder and temperature. The apparent yield stress defined by the $0.2 \%$ offset yield stress is indicated by a filled circle. The disorder dependence of hysteretic behavior is in qualitative agreement with the results of RFIM shown in Fig. 1.

Figs. 3(b), 3(c), 3(f), and 3(g) or Figs. 3(b)-3(e) should be induced, respectively. An elastic dipole should dominate the phase of shuffling as well because these preferential shuffling modes even shrink the octahedron along the tetragonal axis if the shuffling is antiphase. As a result, randomly distributed oxygen atoms in Ti-26Nb (at. \%) soft host lattice act as effective random stress fields (RFs), inducing local martensite regions at random (spatially and orientationally). This scenario explains well why interstitial impurity atoms (oxygen) induce nanodomains and suggests that $\mathrm{Ti}-\mathrm{Nb}-\mathrm{O}$ alloy can be a representative system for the application of RFIM.

\section{B. Disorder-induced hysteresis loop rounding}

Figure 4 shows series of stress-strain $(\sigma-\varepsilon)$ curves of Ti-26Nb- $(0,0.3,0.5,1.0) \mathrm{O}$ (at. \%) alloys at 173, 233, and 293 K. After loading up to around its elastic limit, the tensile stress was removed, and then heated up to $533 \mathrm{~K}\left(\gg T_{\mathrm{c}}^{*}\right)$. For the alloys exhibiting a shape memory effect by the heating, obtained shape recovery strains are indicated by arrows. The apparent yield stress defined by the $0.2 \%$ offset yield stress is indicated by a filled circle. The first feature reveals a change in transformation behavior from shape memory effect to superelasticity with increasing the amount of oxygen. This is because a large decrease in $T_{\mathrm{c}}^{*}$ by oxygen doping. ${ }^{27}$ The other effect of disorder is the shape variation in hysteresis curves. The disorder dependence of hysteretic behavior is in qualitative agreement with that predicted by RFIM shown in Fig. 1. In 00 pure alloy at $293 \mathrm{~K}$, MT takes place at almost constant stress after reaching the critical stress for inducing martensite. Therefore, rectangle-shaped sharp curves with a relatively large hysteresis are formed. In the case of $0 \mathrm{O}$ alloy $\left(T_{\mathrm{c}}^{*} \sim 280 \mathrm{~K}\right)$, the observed nonlinear response at 173 and $233 \mathrm{~K}$ is not due to MT but the martensite domain switching. 


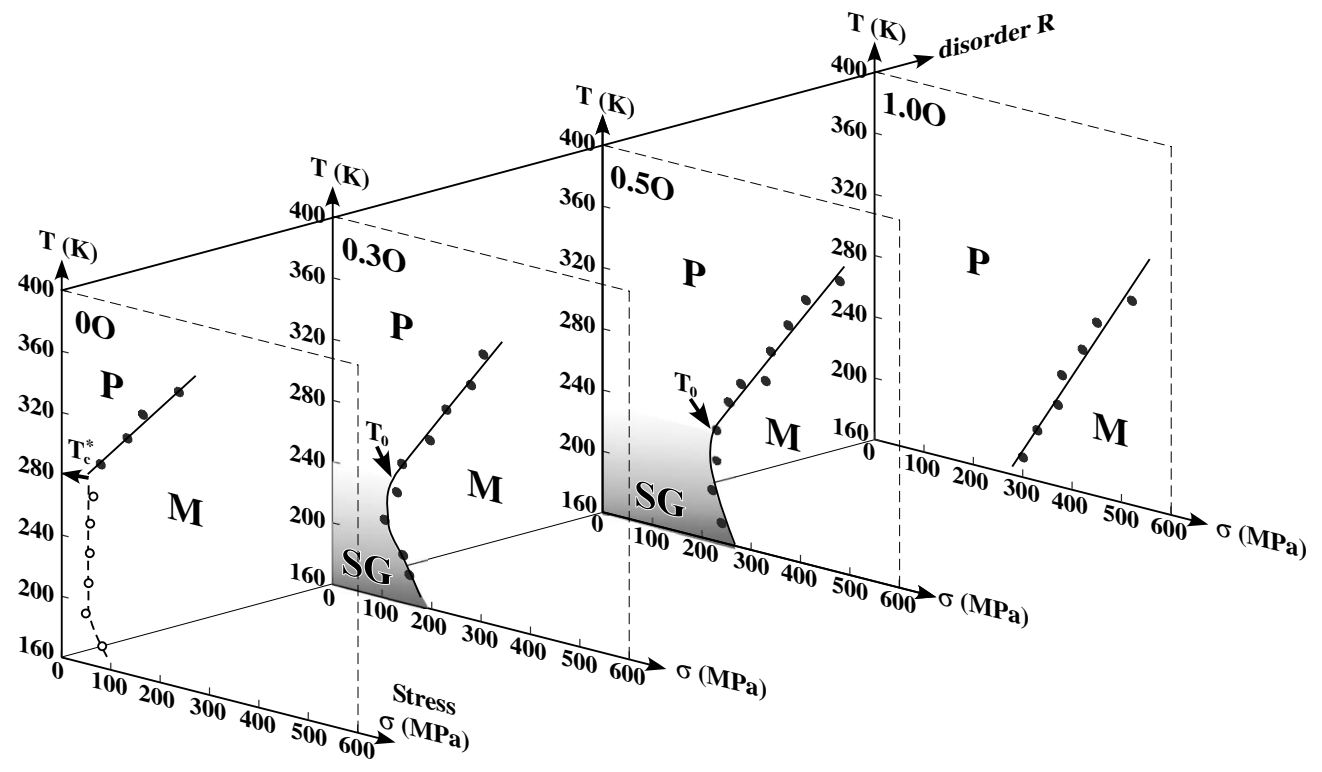

FIG. 5. Stress-temperature-disorder $(\sigma-T-R)$ phase diagram of Ti-26Nb- $x \mathrm{O}$ (at. $\%)$ alloys $(x=0,0.3,0.5,1.0)$. The phase boundaries which separate the cubic parent phase $(\mathrm{P})$ and the orthorhombic martensite phase $(\mathrm{M})$ were obtained by $0.2 \%$ offset yield stress of tensile testing at various temperatures as shown in Fig. 4. Solid lines and broken line are guide to the eyes. Open circles of $0 \mathrm{O}$ alloy correspond to the rearrangement of martensitic domains, separating the multi- and single-martensitic domain states. Dark region of $0.3 \mathrm{O}$ and $0.5 \mathrm{O}$ alloy indicate stain glass phase (SG) (see text).

These behaviors should be therefore excluded in this discussion. With increasing the amount of disorder, there appears rounding of hysteresis loops: the wide and sharp hysteretic behavior changed to slim one with little hysteresis. The stress plateau also disappears. Especially, $\sigma-\varepsilon$ curves for 1.00 alloy at $293 \mathrm{~K}$ shows a nonlinear elastic behavior without any clear yield point, where the apparent Young's modulus continuously decrease with increasing tensile stress. The variation in hysteresis behavior can be understood by RFIM. In case of pure system, ordinary MT process, or nucleation-andgrowth one, is the main mechanism of MT. When the system is loaded up to its critical stress, nucleation of martensite domain occurs in parent domain, and by further loading the domain continues to grow. As a result, phase transformation process is composed of some large avalanches, exhibiting a sharp hysteresis loop with a stress plateau. However, with increasing the amount of disorder, the formation of local stress fields would modify this simple scenario. As confirmed by a dark-field TEM micrograph of 1.00 alloy [Fig. 2(f)], there exist many local martensitelike domains in the cubic parent phase even far above $T_{\mathrm{c}}^{*}$. In the absence of external stress, these domains are spatially and orientationally at random. If an external stress is applied and overcomes its local RFs in some place of the system, the local domain may reorient and grow until it is bound by other local fields. If the RFs are so strong that the reorientation of local domain finishes in a single event without triggering the reorientation of the adjacent domains, the transformation process will be composed of a multitude of the microscopic discontinuities corresponding to a great number of the switching of nanodomains. As a result, when disorder is added, the hysteresis loop becomes slim and round, and would also contribute to the nonlinear elastic behavior where homogeneous reorientation of nanodomains occurs continuously from a low to high stress. The similar idea has already been verified experimentally in another ferroic system, or ferroelectric relaxors, where polar nanoregions (local ordering of electric dipoles) redistribute by the application of an electric field. ${ }^{37}$ This was confirmed by three-dimensional-reciprocal space mapping of diffuse scattering intensity which reflects the distribution of polar nanoregions in real space.

The apparent yield stress derived from tensile testing at various temperatures is plotted as a function of temperature in Fig. 5, giving the stress-temperature-disorder $(\sigma-T-R)$ phase diagram. In the $0 \mathrm{O}$ pure alloy $\left(T_{\mathrm{c}}^{*} \sim 280 \mathrm{~K}\right)$ a critical stress represented by closed circles linearly decreases with decreasing temperature, following Clausius-Clapeyron relationship. These stresses correspond to stress-induced MT. At $280 \mathrm{~K}$ the $0 \mathrm{O}$ alloy undergoes MT, and the critical stress below $280 \mathrm{~K}$ (open circles) corresponds to the rearrangement of martensitic domains. However, by the introduction of oxygen atoms, besides the phase boundary shift toward lower temperature, the critical stress significantly deviates from linear at low temperature, and with further cooling it begins to rise in turn. This behavior can be understood by recently reported strain glass regime. ${ }^{12}$ In the $\mathrm{Ti}_{48.5}-\mathrm{Ni}_{51.5}$ strain glass case, the critical stress decreases with decreasing temperature, obeying Clausius-Clapeyron relationship, whereas it begins to deviate from linear below an ideal freezing temperature $T_{0}$ at which local lattice strain starts freezing. By further cooling, the critical stress rises in turn, disobeying ClausiusClapeyron relationship, which is similar to the behavior of $0.3 \mathrm{O}$ and $0.5 \mathrm{O}$ alloys. The strain glass state can be obtained by sufficient doping of random point defects, which prevents the formation of long-range strain order (martensite) and freezes the local lattice strain in disordered configuration at $T_{\mathrm{g}} \cdot{ }^{9-14}$ In the $\mathrm{Ti}-\mathrm{Nb}-\mathrm{O}$ case the interstitial oxygen atom acts as a strong random point defect. Thus, the observed violation 


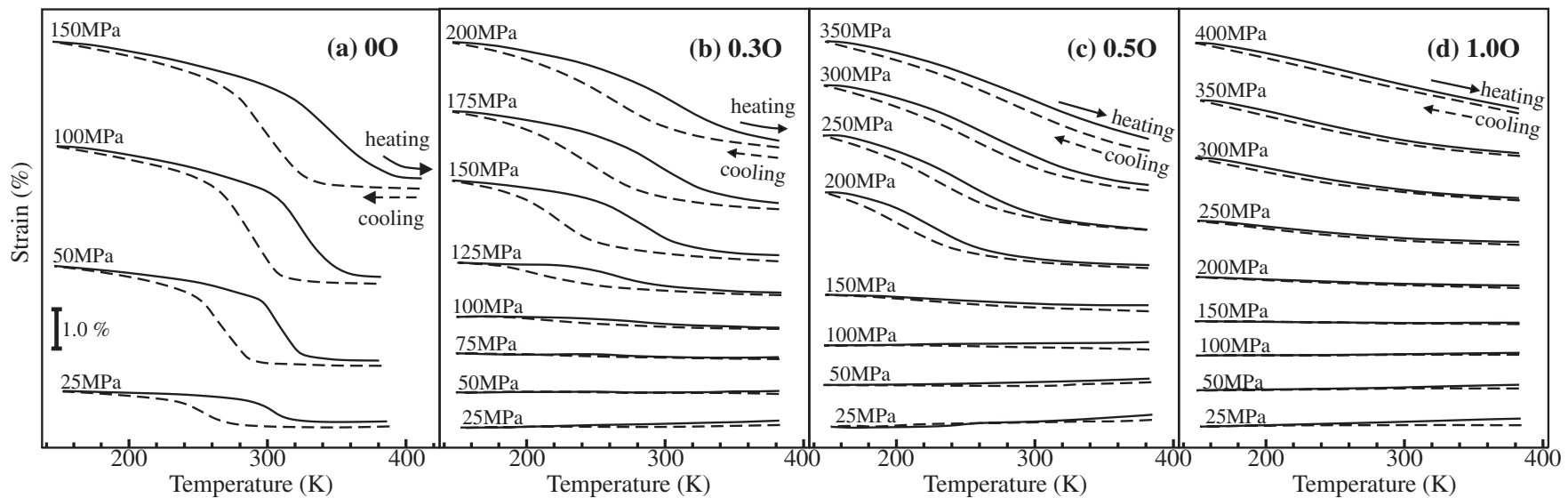

FIG. 6. Strain-temperature curves under various constant stress conditions in several amounts of disorder (a) $x=0 \mathrm{O}$, (b) $x=0.3 \mathrm{O}$, (c) $x=0.5 \mathrm{O}$, and (d) $x=1.0 \mathrm{O}$ for Ti- $26 \mathrm{Nb}-x \mathrm{O}$ (at. \%) alloys. A variation in hysteresis loop is confirmed by varying the amount of disorder.

of the Clausius-Clapeyron relationship in 0.30 and 0.50 alloys is attributable to the transition into a glassy state when cooled below an ideal freezing temperature $T_{0}[\sim 240 \mathrm{~K}$ $(0.3 \mathrm{O})$ and $\sim 230 \mathrm{~K}(0.5 \mathrm{O})]$. (1.0O alloy does not show this anomaly presumably due to insufficient cooling.) In fact, an XRD measurement for the $0.3 \mathrm{O}, 0.5 \mathrm{O}$, and $1.0 \mathrm{O}$ alloys reveals no macroscopic symmetry breaking via cooling from 293 to $133 \mathrm{~K}$, whereas the $0 \mathrm{O}$ pure alloy undergoes MT by cooling (not shown). These results suggest that the obtained shape memory effect of $0.3 \mathrm{O}$ at $173 \mathrm{~K}$ in Fig. 4 may be different from ordinary one, because the starting configuration is a strain glass, but not a twinned martensite, as shown in Fig. 5. In addition, the fact that a single martensitic domain is retained after a removal of the external stress indicates that a strain glass is metastable and has the origin in kinetics.

Recently, the effect of disorder and anisotropy on MT was systematically studied by a Ginzburg-Landau-based model, as a more precise model than the present simple spin-based one. This model also described the disorder-induced rounding of $\sigma$ - $\varepsilon$ curves, ${ }^{38}$ and exhibited glassy phase in highly disordered region successfully, ${ }^{14,39}$ which are in good qualitative agreement with the experimental results obtained here.

MT can be also induced by temperature variation, which provides strain-temperature $(\varepsilon-T)$ hysteresis loop. Figure 6 shows disorder dependence of $\varepsilon-T$ curve under various constant stresses. Solid and broken lines indicate heating and cooling runs, respectively. Due to the experimental limit, the samples were cooled down only to around $150 \mathrm{~K}$, which is not cool enough for $0.5 \mathrm{O}$ and $1.0 \mathrm{O}$ alloys to finish MT. However, we can capture the essence of disorder dependence of the hysteresis loop. The $0 \mathrm{O}$ pure alloy exhibits typical $\varepsilon-T$ curves for shape memory alloy with large hysteresis. The transformation strain increases by increasing constant stress due to the sufficient growth of preferential martensitic domain. With increasing the amount of disorder, the relatively sharp and wide hysteresis loop in $0 \mathrm{O}$ alloy becomes a round and slim one, and the coexistence region between parent and martensite phases arises over a wide range of temperature. In addition, there appears to be a threshold stress below which the transformation strain cannot be confirmed. In $0.5 \mathrm{O}$ alloy, for example, this behavior is clearly observed with a thresh- old stress of $200 \mathrm{MPa}$. It can be understood by recognizing the strain glass phase in oxygen-doped Ti-Nb alloy. As represented in Fig. 5, the critical stress for inducing martensite in $0.3 \mathrm{O}$ and $0.5 \mathrm{O}$ alloys exhibits a minimum at a temperature. Thus when the applied stress is lower than the minimum, the strain glass transition may occur without macroscopic symmetry breaking, whereas normal MT happens if the applied stress is higher than the minimum. The minimum stresses of $0.3 \mathrm{O}$ and $0.5 \mathrm{O}$ alloys are about $100 \mathrm{MPa}$ and 200 $\mathrm{MPa}$, respectively, which is consistent with the threshold stresses in the $\varepsilon$ - $T$ curves.

By converting stress in $\sigma-\varepsilon$ curves to temperature in $\varepsilon-T$ curves, the disorder dependence on hysteresis loop of these $\varepsilon-T$ curves have some similarities to that of $\sigma-\varepsilon$ curves. This suggests that the effect of disorder on $\varepsilon-T$ curves may also be interpreted in the RFIM picture. ${ }^{40}$ In a clean system $(0 \mathrm{O}$ alloy), there is little spatial fluctuation in $T_{\mathrm{c}}^{*}$, as confirmed by dark-field TEM micrographs in Fig. 3(d), and once martensite nuclei come into existence at $T_{\mathrm{c}}^{*}$, they will grow via elastic interaction as a large avalanche. Therefore, the MT of clean system finishes within a narrow temperature range, leading to a sharp hysteresis loop. The small rounding of hysteresis in $0 \mathrm{O}$ alloy may originate from some kind of disorder such as grain boundary and omega phase which is a metastable phase characteristic in Ti-based alloys. ${ }^{26,41}$ In a dirty system, there is large spatial fluctuation in $T_{\mathrm{c}}^{*}$. Even far above apparent $T_{\mathrm{c}}^{*}$, local strain order may develop at some regions with strong RFs, which is confirmed as nanodomains by TEM images. The application of an external tensile stress can yield a macroscopic strain due to the rearrangement of those nanodomains. However, the nucleated or reoriented domains are prevented from growing by disorder-induced pinning, leading to small avalanches. Thus it is necessary to cool far below apparent $T_{\mathrm{c}}^{*}$ so as to percolate the whole system (complete MT). As a result, the $\varepsilon$ - $T$ hysteresis loop becomes round and slim. In fact, the observed slim loops of $1.0 \mathrm{O}$ alloy are well explained by a series of tiny nanodomain generation and reorientation.

\section{SUMMARY AND CONCLUSIONS}

In summary, we have investigated the effect of disorder on MT behavior, especially on hysteretic behavior of disor- 
dered Ti-26Nb- $x \mathrm{O}$ (at. \%) alloys $(x=0,0.3,0.5,1.0)$. This system allows arbitrary tuning of the amount of disorder by the introduction of interstitial oxygen atoms into elastically soft host lattice Ti-26Nb $\left(T_{\mathrm{c}}^{*} \sim 280 \mathrm{~K}\right)$. Dark-field TEM images from superlattice spot originating from martensite directly prove that interstitial oxygen atoms induce nanometersized martensite (nanodomain) at random (spatially and orientationally) despite the sharp decrease in $T_{\mathrm{c}}^{*}$ : with increasing the amount of disorder, nanodomains grow in size and number, and eventually spread throughout the specimen for Ti-26Nb-1.0O alloy. From both stress-strain and straintemperature curves, a clear variation in hysteresis loops from a sharp and wide to a round and slim one were confirmed with the increment of oxygen content (disorder). These results are in qualitative agreement with that predicted by RFIM. From the microscopic point of view, MT accompanied by shuffling can elongate the distance between the nearest-neighbor atoms from the interstitial oxygen, relaxing the internal strain energy, dependent on which martensitic domains are induced. The agreement between experimental results and RFIM indicates that the MT picture in strong disorder regime should differ substantially from the ordinary nucleation-and-growth process, where quite small size of matensites homogeneously generate and percolate the whole system.

\section{ACKNOWLEDGMENTS}

Y.N. would like to thank Y. Wang, P. J. Buenconsejo, Y. W. Chai, Y. Al-Zain, M. Tahara, and H. Tobe for fruitful discussions. This work was partially supported by ILC Project from University of Tsukuba and the Grants-in-Aid for Fundamental Scientific Research [Kiban A(2002-2004), Wakate B(2006-2007), and Kiban C(2008-2010)] from the Ministry of Education, Culture, Sports, Science and Technology. This work was also partially supported by WCU (World Class University) program through the National Research Foundation of Korea funded by the Ministry of Education, Science and Technology (Grant No. R32-2008-000-20093$0)$.
${ }^{1}$ Shape Memory Materials, edited by K. Otsuka and C. M. Wayman (Cambridge University Press, Cambridge, 1998).

${ }^{2}$ J. P. Sethna, K. A. Dahmen, S. Kartha, J. A. Krumhansl, B. W. Roberts, and J. D. Shore, Phys. Rev. Lett. 70, 3347 (1993).

${ }^{3}$ Y. Yamada, Mater. Trans., JIM 33, 191 (1992).

${ }^{4}$ L. Carrillo and J. Ortín, Phys. Rev. B 56, 11508 (1997).

${ }^{5}$ E. Vives, J. Ortín, L. Mañosa, I. Ràfols, R. Pèrez-Magranè, and A. Planes, Phys. Rev. Lett. 72, 1694 (1994).

${ }^{6}$ X. Ren, Y. Wang, Y. Zhou, Z. Zhang, D. Wang, G. Fan, K. Otsuka, T. Suzuki, Y. Ji, J. Zhang, Y. Tian, S. Hou, and X. Ding, Philos. Mag. 90, 141 (2010).

${ }^{7}$ K. Binder and A. P. Young, Rev. Mod. Phys. 58, 801 (1986).

${ }^{8}$ G. A. Samara, J. Phys.: Condens. Matter 15, R367 (2003).

${ }^{9}$ S. Sarkar, X. Ren, and K. Otsuka, Phys. Rev. Lett. 95, 205702 (2005).

${ }^{10}$ Y. Wang, X. Ren, and K. Otsuka, Phys. Rev. Lett. 97, 225703 (2006).

${ }^{11}$ Y. Wang, X. Ren, K. Otsuka, and A. Saxena, Phys. Rev. B 76, 132201 (2007).

${ }^{12}$ Y. Wang, X. Ren, K. Otuska, and A. Saxena, Acta Mater. 56, 2885 (2008).

${ }^{13}$ Y. Wang, X. Ren, and K. Otsuka, Mater. Sci. Forum 583, 67 (2008).

${ }^{14}$ X. Ren, Y. Wang, K. Otsuka, P. Lloveras, T. Castán, M. Porta, A. Planes, and A. Saxena, MRS Bull. 34, 838 (2009).

${ }^{15}$ Y. Imry and M. Wortis, Phys. Rev. B 19, 3580 (1979).

${ }^{16}$ J. P. Sethna, K. A. Dahmen, and C. R. Myers, Nature (London) 410, 242 (2001).

${ }^{17}$ K. A. Dahmen and J. P. Sethna, Phys. Rev. B 53, 14872 (1996).

${ }^{18}$ F. J. Pérez-Reche and E. Vives, Phys. Rev. B 67, 134421 (2003).

${ }^{19}$ F. J. Pérez-Reche, M. L. Rosinberg, and G. Tarjus, Phys. Rev. B 77, 064422 (2008).

${ }^{20}$ M. Müller and A. Silva, Phys. Rev. Lett. 96, 117202 (2006).

${ }^{21}$ E. Vives and A. Planes, Phys. Rev. B 63, 134431 (2001).

${ }^{22}$ E. Vives and A. Planes, Phys. Rev. B 50, 3839 (1994).

${ }^{23}$ E. Vives, J. Goicoechea, J. Ortín, and A. Planes, Phys. Rev. E
52, R5 (1995).

${ }^{24}$ B. Cerruti and E. Vives, Phys. Rev. B 77, 064114 (2008).

${ }^{25}$ M. L. Rosinberg and T. Munakata, Phys. Rev. B 79, 174207 (2009).

${ }^{26}$ H. Y. Kim, Y. Ikehara, J. I. Kim, H. Hosoda, and S. Miyazaki, Acta Mater. 54, 2419 (2006).

${ }^{27}$ S. Miyazaki, H. Y. Kim, and H. Hosoda, Mater. Sci. Eng., A 438-440, 18 (2006).

${ }^{28}$ A. S. Nowick and B. S. Berry, Anelastic Relaxation in Crystalline Solids (Academic, New York, 1972), Chap. 8 and 9.

${ }^{29}$ Y. Murakami and D. Shindo, Philos. Mag. Lett. 81, 631 (2001).

${ }^{30}$ S. Kartha, J. A. Krumhansl, J. P. Sethna, and L. K. Wickham, Phys. Rev. B 52, 803 (1995).

${ }^{31}$ L. E. Tanner, D. Schryvers, and S. M. Shapiro, Mater. Sci. Eng., A 127, 205 (1990).

${ }^{32}$ S. Muto, S. Takeda, R. Oshima, and F. E. Fujita, J. Phys.: Condens. Matter 1, 9971 (1989).

${ }^{33}$ P. Lloveras, T. Castán, M. Porta, A. Planes, and A. Saxena, Phys. Rev. Lett. 100, 165707 (2008).

${ }^{34}$ D. E. Jiang and E. A. Carter, Phys. Rev. B 67, 214103 (2003).

${ }^{35}$ L. Yu, F. Yin, and D. Ping, Phys. Rev. B 75, 174105 (2007).

${ }^{36}$ A. R. G. Brown, D. Clark, J. Eastabrook, and K. S. Jepson, Nature (London) 201, 914 (1964)

${ }^{37}$ G. Xu, Z. Zhong, Y. Bing, Z.-G. Ye, and G. Shirane, Nature (London) 5, 134 (2006).

${ }^{38}$ P. Lloveras, T. Castán, M. Porta, A. Planes, and A. Saxena, Phys. Rev. B 81, 214105 (2010).

${ }^{39}$ P. Lloveras, T. Castán, M. Porta, A. Planes, and A. Saxena, Phys. Rev. B 80, 054107 (2009).

${ }^{40}$ If an external constant stress is not high enough to lift the degenerate states, RFPM (Ref. 24) would be more desirable one for the precise description of temperature-induced MT, since temperature is a scalar external field, leading to multidomain state.

${ }^{41}$ D. De Fontaine and R. Kikuchi, Acta Metall. 22, 1139 (1974). 